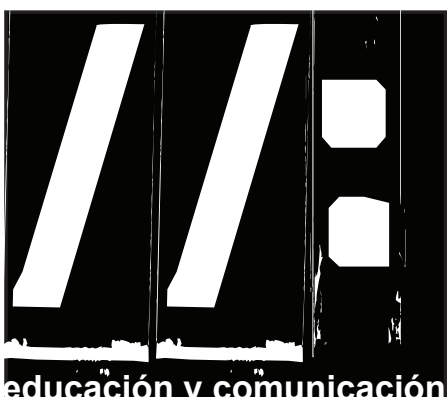

15:95-100 Nov. 2017

\section{LA DIVERSIDAD AFECTIVO SEXUAL Y DE IDENTIDAD DE GÉNERO EN EI MARCO DE LA ATENCIÓN A LA DIUERSIDAD}

\section{Sexual affective diversity and gender identity in the framework of the attention to diversity}

\author{
Fducación COGAM/Universidad Autónoma de A. M. Vela. \\ E.mail: Josevela.genero@gmail.com
}

Resumen:

La sociedad es diversa y una representación de la misma se da reúne en las aulas de los centros educativos. La importancia de la atención a la diversidad se fundamenta en los beneficios de una educación preparada para formar al alumnado en la variedad de la población española. La implementación de estas perspectivas mejoran la implicación con las materias y, por ello, mejora el rendimiento académico, alejando el riesgo de exclusión al que se enfrentan los individuos que se alejan demasiado del modelo normativo. La población de lesbianas, gais, bisexuales y transexuales también está representada en nuestras aulas y requiere de un acercamiento a sus necesidades como cualquier otra particularidad del alumnado.

Palabras clave educación, atención a la diversidad, LGBT.

\title{
Abstract:
}

The society is diverse and a representation of it is given meets in the classrooms of the educational centers. The importance of attention to diversity is based on the benefits of an education prepared to train students in the variety of the Spanish population. The implementation of these perspectives improves the involvement with the subjects and, therefore, improves the academic performance, taking away the risk of exclusion faced by individuals who are too far from the normative model. The lesbian, gay, bisexual and transgender population is also represented in our classrooms and requires an approach to their needs like any other particularity of the students.

Word Keys: education, attention to diversity, LGBT.

Recibido 16-06-2017 / Revisado 13-07-2017 / Aceptado 19-08-2017 / Publicado 01-11-2017 


\section{II: Diversidad y educación afectivo sexual}

\section{Interés y pertinencia de la atención a la diversidad}

-1 profesorado es responsable en gran parte de las Cinámicas de aprendizaje y en sus manos se encuentran muchas de las claves para que el alumnado logre los objetivos del curso y promocione adecuadamente (Salazar 2007:27-50). La atención a la diversidad es una condición transversal para un ejercicio profesional de la docencia. Responde a la necesidad de hacer llegar la educación al mayor porcentaje posible de la población. La atención efectiva a la diversidad entre los deberes de los y las docentes tanto por su dimensión normativa de obligado cumplimiento (LOMCE 8/2013 de 9 de diciembre; UNESCO, 1995) como por la necesidad práctica de su correcta aplicación, como veremos a continuación.

La diversidad como reto docente es frecuentemente eclipsado por otras perspectivas. Con frecuencia oímos al profesorado eso de: antes se estudiaba mejor, olvidando que a veces, ese antes era cuando solo lo hacía una minoría de la población (Martínez García, 2005). Sin embargo, se trata de que unos pocos sigan teniendo buenos resultados académicos sin perder a aquellos que pueden tenerlos menos buenos. Para ello es necesaria la inversión en enseñanza: profesionalidad, preparación de docentes, formación continua, evaluaciones y dar cuenta de la diversidad adaptándonos a las necesidades específicas del aula y del alumnado (Feito, 2002). Reconociendo, al tiempo, la importancia del currículo oculto que porta cada alumno o alumna (Connell 1997a). La cultura de la educación es la cultura sociopolítica de la clase media (Feito, 2007) y la heteronormatividad (Moreno, 2006). La clase media, su ética, lenguaje y cultura, así como las familias nucleares heterosexuales, son el modelo do- minante en educación, que privilegia unos modelos, (Pumares, y Hernández, 2007) que son tomado por el alumnado como referentes normativos.

En la práctica la no atención a las peculiaridades del alumnado conlleva el desinterés, la falta de capacidad comprensiva, o, incluso, la incapacidad de respuesta. Adicionalmente, apreciar las diferencias e incorporar este aprecio, junto con el aprendizaje de los derechos humanos, en el modelo educativo es de máxima importancia. Y dado el alto nivel de agresiones, acoso y ciberbullying que se dan en los centros queda patente que no estamos sabiendo cubrir esta necesidad (Defensor del Pueblo 2007; COGAM, 2016; ; COGAM, 2016b).

\section{¿Qué entendemos por diversidad?}

El concepto de diversidad es un poco más amplio y podríamos podríamos estar implementándola de manera descuidada (Sánchez, 2013). Las diferencias son más evidentes en algunos casos y más sutiles en otros. Detectar un comentario desafortunado sobre diversidad puede ser en éste último caso más difícil que en el primero. Pero en ambos tendrá consecuencias negativas.

Para trabajar el concepto de diversidad podemos hacer una clasificación con la intención de sistematizar un mayor espectro y gracias a ello poder desarrollar herramientas funcionales. Siguiendo las categorías propuestas por Mercedes Sánchez (2013), podemos establecer un abanico de cuatro grupos: Diversidades biológicas, funcionales, culturales y sexuales. Sin embargo algunas de estas categorías son tradicionalmente más atendidas de lo que, al menos en materia de acoso, se previene en los centros (Pichardo, 2015). 


\section{1/: La diversidad afectivo sexual y de identidad de}

Siendo la categoría de diversidad afectivo-sexual y de identidad de género, la que más acoso recibe, nos centraremos en desarrollarla con más detalle.

\section{Diversidad biológica}

Comenzamos por la diversidad biológica, según la cual entendemos las diferencias que se dan dentro de los cuerpos en referencia al modelo normalizado. Dentro del alumnado existen diferencias físicas en base a las cuales se pueden llegar a estigmatizar a algunos individuos. Diferentes cuerpos tienden a estar minusvalorados, sobre todo por el referente publicitario de cuerpos blancos y esculturales. Dentro de esta diversidad incluimos modelos raciales, ausentes también de los imperantes cánones de belleza. Quizá sea necesario aclarar que el concepto de raza es empleado en su sentido cultural pues la existencia de razas dentro del ser humano es una idea desterrada de las ciencias sociales (Kottak, 2011: 143). El problema se agudiza cuando los medios educativos: materiales, recursos didácticos y manuales de materia; continúan reproduciendo un único físico estándar, olvidando representar las realidades del aula.

\section{Diversidades funcionales}

Siguiendo el pensamiento de Palacios y Romañach (2006) las personas con discapacidad lo son en cuanto a comparadas con un modelo ideal, el rango de discapacidad responde a la distancia con este modelo que no se construye matemáticamente sino, de nuevo, respecto a unos modelos normativos. Esta diversidad suele estar más visibilizada en las normativas y planificaciones educativas institucionales. La implemen- tación de la atención a la diversidad pasa, en muchas ocasiones, por la eliminación de barreras de movilidad, mobiliario adaptado y equipos de apoyo.

\section{Diversidad cultural}

No olvidamos que el alumnado perteneciente a distintas culturas escolarizado debe tener igual acceso a la educación. Las particularidades culturales pueden distanciar de la lógica didáctica mayoritaria y, por ello, alejar al alumnado de la educación. Lo que conlleva, recordamos, a aumentar las posibilidades de exclusión. La inclusión de las comunidades culturales en el sistema educativo dependen, en primera instancia, de la implicación político-administrativa. Sin embargo, dentro del cuerpo docente, es necesario eliminar las perspectivas colonialistas y el etnocentrismo cultural para acercar a las diversas comunidades culturales a la materia.

\section{Diversidad afectivo-sexual $y$ de identidad de género}

Por último, nos centramos en los adolescentes, lesbianas, gais, bisexuales y trans* (LGBT). Estas categorías se convierten en diversidades al entrar en conflicto con ideologías normativas de la sexualidad que pretender ordenar la sociedad en torno a una moral determinada. Olvidando con frecuencia el superior interés del menor que legalmente se señala a nivel nacional (LOMCE 8/2013) e internacional (UNESCO 2016).

Se tiende en los institutos a trabajar exclusivamente modelos heterosexuales como los únicos posibles, llegando a omitir otras realidades sexo-afectivas. Por 
el contrario se vienen constatando en los institutos españoles que alrededor de un $10 \%$ de los jóvenes no se consideran heterosexuales y un $16 \%$ duda o no tiene clara su orientación sexual (Pichardo, 2009). Para estos jóvenes el modelo heterosexual no es uno con el que se sientan identificados y, además, son los que sufren el mayor nivel de acoso dentro de nuestros centros educativos (Generelo, 2005; Pichardo, 2015). El alto nivel de estrés sufrido por su orientación sexual o identidad de género les empuja especialmente a plantearse no solo el abandono escolar, sino el suicidio (Takás, 2006; Generelo, 2012). Todo esto señala la importancia de tener en cuenta las necesidades del alumnado LGBT.

\section{Especificidad de la atención a la diversidad para alumnado LGBT}

Este alumnado necesita que se incorpore su realidad como medida para prevenir procesos de exclusión pues sufren un tipo específico de acoso llamado LGBT-fobia (anteriormente denominada homofobia). Los menores crecen junto a dos insultos recurrentes a lo largo de su vida. Estos son: maricón, para los chicos y puta, para las chicas. A las chicas lesbianas se las ofende con el término bollera (Platero, 2010).

La especificidad de la problemática LGBT se distingue en los siguientes elementos. El alumnado LGBT ha de revelar su particularidad y abandonar la normalidad para desarrollar una identidad relacional sana. Es decir, para no vivir una mentira han de manifestarse como LGBT pues de otro modo se les asumiría cis-heteros. Esto es causa de la heteronormatividad (Warner, 1993, Moreno, 2006). Este acto de revelación ha de realizarse también en el hogar, por lo que, con frecuencia, carecen del apoyo familiar previo. Frecuentemente temen tanto la reacción de su familia como la de amistades y compañeros. Para evitar confrontar esta situación llegan a aceptar cualquier tipo de acoso y vejación (Generelo, 2008) evitando así la intervención de profesores y familia (Educación-COGAM 2013; 2015).

Otro de los elementos específicos de la diversidad LGBT que no comparten otras diversidades es la noción de contagio del estigma (Goffman, 1989). En espacios LGBT-fóbicos, todo aquel que decida ser amigo o proteja a un compañero insultado como gay y aquella que haga lo propio con una compañera señalada como lesbiana, se arriesga a ser así mismo a ser excluido como gay o lesbiana. Este proceso agrava el nivel de exclusión de lesbianas, gais, bisexuales y jóvenes trans* pues, por un lado dificulta el acercamiento de los pares, y por otro, genera en el alumno o alumna la culpa de transmitir el acoso a quienes aprecia.

Por otro lado, en ocasiones, gracias a la sensibilización contra la LGBT-fobia, los centros educativos se convierten en espacios seguros frente a familias y entornos intolerantes (COGAM, 2016) permitiendo al alumnado madurar y adquirir competencias para orientar su futuro de manera adecuada.

\section{La diversidad de género y el alumnado trans*}

Mención a parte, dentro de la diversidad LGBT, merece el alumnado trans*, si bien sería necesario un bibliografía específica (Platero, 2014), podemos esbozar un par de párrafos aclaratorios. Las personas trans* son aquellas que tienen una identidad de género (es decir, chico o chica) distinta a aquella con la fueron 


\section{1/: La diversidad afectivo sexual y de identidad de}

definidas al nacer. Para simplificar, a una criatura, según sus genitales se le asignó un género con el que no se siente cómoda, pudiendo demandar un tratamiento distinto al que teníamos previsto oficialmente.

La adolescencia supone un momento importante en la asunción de la identidad de género (Shaffer, 2000), por lo que es necesario evitar generar situaciones que pueden llegar a ser innecesariamente traumatizantes según como el centro las resuelva. Baños mixtos, uniformes unisex o listas basadas en los apellidos eliminan barreras eficazmente.

Es interesante señalar que las personas trans* también tienen una orientación afectivo-sexual. Pueden ser heterosexuales, homosexuales y bisexuales como las personas cisexuales. Por otro lado, las consideraciones sobre la anatomía de las personas trans* son estrictamente privadas. Adicionalmente aclaramos que el término hermafroditismo no es correcto al aplicarse en el ser humano. Existen personas intersexuales, individuos que presentan un síndrome biológicos por el cuales, simplificando enormemente, su sexo cromosómico no coincide con la genitalidad o género asignado socialmente (Gregori, 2006).

\section{La docencia y la diversidad}

Los docentes han de comprender que existe una variedad poblacional que queda representada en el aula. Una efectiva atención a la diversidad acerca la materia al alumnado y esto mejora el rendimiento. Para ello, en palabras de Muñoz Garrido (2007): "La mejora de la calidad educativa solo es posible si se da un proceso permanente de profesionalización pedagógica de los docentes".

La atención a la diversidad y la sensibilidad con la diferencia funcionan en tres niveles. Primero, la atención a diversos ritmos de trabajo es necesaria para permitir una aprendizaje correcto dentro del aula (Rodríguez, 2007). En segundo lugar, la relación entre mejora de técnicas docentes y disminución de las tasas de fracaso escolar es manifiesta (Echeita, 2006). Por último, el uso de dinámicas inclusivas en el aula y la inclusión dentro del modelo educativo acerca la materia de trabajo a la realidad de cada individuo al tiempo que fomenta la transmisión de estos mismos valores. La inclusión, la tolerancia, la valoración de la diversidad social, son parte del proceso de maduración necesarios para vivir en sociedad.

\section{Referencias}

COGAM; López, Ekaitz (Coord.) (2013). Homofobia en las aulas. Madrid: Grupo de Educación de COGAM.

Disponible en: http://www.cogam.es/secciones/educacion/documentos-educativos)

COGAM; Benítez, E. (Coord.) (2016a). LGBT-fobia en las aulas 2015. ¿Educamos en la diversidad afectivo-sexual? Madrid: Grupo de Educación de COGAM. Disponible en: https://cogameduca.wordpress.com/ category/investigacion-2014-2016/

COGAM; Benítez, E. (Coord.) (2016b). Ciberbullying LGBT-fóbico. Nuevas formas de intolerancia. Madrid, Grupo de Educación de COGAM.

Disponible en: https://cogameduca.files.wordpress. com/2016/03/4-ciberbullying-lgbt-fc3b3bico-informe-completo-web.pdf

Connell, R. (1997a). Escuelas y justicia social. Madrid: Morata.

Connell, R. (1997b). La organización social de la 
masculinidad. En Valdés, T. y Olavarría, J (Eds.). Masculinidades, poder y crisis. Chile: Flasco.

Defensor del Pueblo. (2007). Violencia Escolar: El maltrato entre iguales en la educación secundaria obligatoria 1999-2006. Madrid: Defensor del Pueblo. Echeita, G. (2006). Educación para la inclusión o educación sin exclusiones. Madrid: Lavel.

Feito, R. (2002). Una educación de calidad para todos. Madrid: Siglo Veintiuno.

Feito, R. (2007). Atención a la diversidad. Clase, etnia y género. En Pumares L. y Hernández, L. (Coords.). La formación del profesorado para la atención a la diversidad. Madrid: CEP.

Generelo, J. y Picharlo, J. I. (2005). Homofobia en el sistema educativo. Madrid: COGAM. Disponbible en :

(http://www.researchgate.net/publication/235349407_Homofobia_en_el_sistema_educativo) (7-11-2015).

Generelo, J.; Pichardo, J. I. y; Galofré, Guillem (2008). Adolescencia y sexualidades minoritarias. Jaén: Alcalá Grupo Editorial.

Generelo, J. (Coord.) (2012). Acoso escolar homofóbico y riesgo de suicidio en adolescentes y jóvenes LGB. Madrid: COGAM/FELGTB

Goffman, E. (1989): Estigma. La identidad deteriorada. Buenos Aires: Amorrortu.

Gregori, N. (2006, enero-febrero). Los cuerpos ficticios de la biomedicina, el proceso de construcción del género en los protocolos médicos de asignación de sexo en bebé intersexuales. AIBR. Revista de Antropología Iberoamericana, 1; 103-124

Kottak, C. P. (2011). Antropología Cultural. Nueva York: Mc Graw Hill.

LOMCE Ley Orgánica 8/2013, de 9 de diciembre, para la mejora de la calidad educativa. Boletín Oficial del Estado 10 de diciembre de 2013. 12886. Disponible en: (https://www.boe.es/buscar/pdf/2013/BOE-A2013-12886-consolidado.pdf)

Martínez García, J. S. (2005). Dos reflexiones sobre el sistema educativo español: el nivel educativo no cae y las clases sociales sí existen. El Viejo Topo, 213. Moreno, Á. y Pichardo, I. (2006). Homonormatividad y existencia sexual. Amistades peligrosas entre género y sexualidad. AIBR. Revista de Antropología Iberoamericana, 1;143-156

Muñoz Garrido, V. (2007). La formación del profesorado para atender a la diversidad: Proteger La Infancia, Prevenir Riesgos. En Pumares L. y Hernández M. L. Formación del profesorado para la atención a la diversidad. Madrid: CEP.

Palacios, Y. y Romañach, J. (2006). El Modelo de la Diversidad: La Bioética y los Derechos Humanos para alcanzar la plena dignidad en la diversidad funcional. España: Diversitas Editorial.

Pichardo, J. I. (2006). Especificidad del acoso escolar por homofobia. En Generelo, J. y Pichardo Galán, J. I. (Coord.) (2006). Homofobia en el sistema educativo. Madrid: COGAM.

Pichardo, J. I. (2009): Adolescentes ante la diversidad sexual. Madrid: Catarata.

Pichardo, J. I. (2015). Abrazar la diversidad. Propuestas para una educación libre de homofobia y transfobia. Instituto de la mujer y para la igualdad de oportunidades. Disponible en:

http://www.inmujer.gob.es/actualidad/NovedadesNuevas/docs/2015/Abrazar_la_diversidad.pdf) (259-2015)

Platero, R. (Lucas) (2010). Estrategias de afrontamiento frente al acoso escolar: una mirada sobre 


\section{1/: La diversidad afectivo sexual y de identidad de Iénero en el marco de la atención a la diversidad}

\section{Divulgatio}

las chicas masculinas. En LES Online, vol. 2, no 2: 35 $\square$ 51. Disponible en: http://www.lespt.org/lesonline/index.php?journal $=$ lo\&page $=$ article \&op $=$ view \&p $\operatorname{ath}[]=36 \&$ path []$=35$ )

Platero, R. (Lucas) (2014). Trans*exualidades. Acompañamiento, factores de salud y recursos educativos. Barcelona: Bellaterra.

Pumares, L. y Hernández, L. (2007). La formación del profesorado para la atención a la diversidad. Madrid: CEP.

Rodríguez, J. A. (2007). "La diversidad del alumnado por diferentes capacidades y ritmos de aprendizaje". En Pumares L. y Hernández, L. (Coords.). La formación del profesorado para la atención a la diversidad. Madrid: CEP.

Shaffer, D. (2000). Desarrollo social y de la personalidad. Madrid: Thomson.

Sánchez Sáinz, M. (2013). Inclusión Educativa. Una Cuestión Pendiente. Madrid: Catarata.
Takács, J. (2006). Social exclusion of younglesbian, gay, bisexual and transgender (LGBT) people in Europe. Brussels: ILGA-Europe. Disponible en https://www.salto-youth.net/downloads/4-1948/ReportSocialExclusionIGLYOilga.pdf? (15-9-1015) UNESCO (1995). Informe final. Conferencia Mundial sobre necesidades educativasespeciales: acceso y calidad. Salamanca, España, 7-10 de junio de 1994. Madrid: MEC.

UNESCO (2016). Out in the open. Education sector responses to violence based on sexual orientation and gender idnetity/expression. Paris: United Nations Education,

Scientific and Cultural Organization. Disponible en: http://www.unesco.org/open-access/terms-use-ccbysa-en)

Warner, M. (1993). Fear of a Queer Planet: Queer Politics and Social Theory. Minneapolis: University of Minnesota Press. 